\title{
« Voilà le moment fatal » : réflexions sur la brièveté formelle et temporelle dans les Mémoires du cardinal de Retz
}

\author{
Louis Laliberté-Bouchard \\ Université McGill
}

La Fronde, soit la guerre civile qui a marqué la régence d'Anne d'Autriche pendant la minorité de Louis XIV pour tenter de déloger le cardinal Mazarin - ultime tentative pour s'opposer à l'absolutisme qui avait commencé à s'instaurer sous Richelieu -, a suscité un grand nombre de Mémoires. Quelques décennies après les troubles (1648-1653), ceux qui y ont pris part - et surtout, dans le cas des mémorialistes, ceux qui en ont été les perdants - désirent faire le point sur cette époque déjà lointaine afin de la comprendre en y jetant un dernier regard rétrospectif. En effet, s'il y a bien une caractéristique qui va de soi en ce qui a trait à l'écriture des Mémoires sous l'Ancien Régime, c'est que leurs rédacteurs se considèrent en mesure 
d'interpréter leur existence, de donner sens à ce qu'ils ont vu, que ce soit en tant qu'acteurs ou témoins. Plus encore, les mémorialistes du XVII ${ }^{e}$ siècle cherchent à dévoiler ce que ceux qui ont écrit la grande histoire voudraient garder dans l'ombre afin de la rectifier, faute d'avoir été justement traités par elle. Or ce rapport à l'histoire et aux évènements est présenté dans une conjoncture unique dans les Mémoires du cardinal de Retz, rédigés en quelques mois vers 1675. En retraçant les principales étapes de son existence, Retz esquisse une histoire qui semble opaque, inexplicable. Le fil qui permettait de la comprendre se rompt, et même s'émiette dans une succession d'instants qui laisse le cardinal dans une profonde obscurité.

Pourtant, l'homme mêlé aux conjurations, complots, partis et factions en tout genre n'en est pas moins dispensé d'agir, et plus encore, de bien agir. De fait, Retz se présente en tant qu'homme d'action, posture qui s'oppose à ceux qui se contentent de la spéculation (Retz, 1987, I, p. 364). C'est ainsi l'expérience vécue au cœur des évènements qui légitime non seulement le témoignage des Mémoires, mais encore les leçons que le cardinal souhaite transmettre.

Pour répondre au problème de l'action, ce sont deux formes brèves que Retz convoque : d'une part les maximes et autres formes de sentences et, d'autre part, les portraits, en ce que les premières permettent de guider sa conduite et les seconds de connaître le caractère d'un être, c'est-à-dire ce qui révèle la nature de sa personnalité dans le but d'en comprendre les comportements. Cependant, conscient que ces deux formes brèves n'offrent qu'une solution bien partielle au problème de l'action (puisque ses maximes et ses observations psychologiques n'ont pas suffi à garantir la victoire à son parti), Retz propose une réponse d'un ordre bien différent du premier. Il substitue l'honneur à la victoire effective, révélant ainsi qu'il y a de la grandeur même dans l'échec, et que l'important est d'avoir su se montrer résolu dans l'adversité, d'avoir tout fait pour saisir les occasions. 


\section{De la critique des historiens et gazetiers à l'opacité du présent}

C'est la critique des historiens et gazetiers présente dans les Mémoires qui permet d'abord et avant tout de constater comment l'histoire, dans toute sa complexité, se joue dans des instants brefs, souvent indéchiffrables et inextricables. Nous verrons que cette critique, plus que de souligner la vanité des écrivains, met en lumière l'obscurité dans laquelle doit évoluer l'homme d'action, car ce qui rend le passé impénétrable à ceux qui veulent l'expliquer pèse encore plus sur ceux qui l'ont fait.

En excluant leur flatterie à l'égard du pouvoir, les gazetiers et les historiens sont blâmés pour deux raisons ${ }^{1}$. La première est qu'ils simplifient les choses en inventant cohérence et raison au fil des évènements :

Ne doit-on pas admirer, après cela, l'insolence des historiens vulgaires, qui croiraient se faire tort si ils laissaient un seul événement dans leurs ouvrages, dont ils ne démêlassent pas tous les ressorts, qu'ils montent et qu'ils relâchent presque toujours sur des cadrans de collège? (Retz, 1987, II, p. 155)

À la machine bien réglée mais factice des historiens répond plutôt la métaphore organique d'un « corps monstrueux et presque incompréhensible» (Retz, 1987, II, p. 303) chez le cardinal qui engendre une « quasi-impossibilité du genre historique » (Garapon, 2011, p. 130).

De même, Retz critique, dans une formule similaire, la prétention des historiens et gazetiers à déceler les motifs psychologiques de ceux qui ont fait l'histoire :

J'admire à ce propos l'insolence de ces gens de néant en tout sens, qui, s'imaginant d'avoir pénétré dans tous les replis des cœurs de ceux qui ont eu le plus de part dans ces affaires, n'ont laissé aucun événement dont ils n'aient prétendu avoir développé l'origine et la suite (Retz, 1987, II, p. 159-160).

\footnotetext{
${ }_{1}^{1}$ Précisons d'emblée notre dette envers les travaux de Frédéric Charbonneau (p. 77-86) et de Emmanuèle LesneJaffro (p. 489-493) sur cette question du rapport de Retz aux gazetiers et historiens.
} 
Ainsi, la complexité du récit de Retz, qui va jusqu'à la confusion au fur et à mesure que la Fronde s'étiole ${ }^{2}$, est la conséquence logique d'une conception de l'histoire qui ne peut céder à la simplification. Quand elle se ramifie, l'œuvre doit aussi en témoigner.

L'imagination de ces petits esprits qui prennent « le vraisemblable pour le vrai » (Retz, 1987, I, p. 322) nous mène à la deuxième critique du cardinal, critique qui découle naturellement de la première. Puisque le réel est si complexe, seuls ceux qui y ont participé sont en mesure d'en rendre compte. Or ce n'est pas le cas des gazetiers et historiens :

Je vous supplie de me permettre que je fasse ici une pause, pour observer qu'il n'est pas étrange que les historiens qui traitent des matières dans lesquelles ils ne sont pas entrés par eux-mêmes s'égarent si souvent, puisque ceux même [sic] qui en sont les plus proches ne se peuvent défendre, dans une infinité d'occasions, de prendre pour des réalités des apparences quelquefois fausses dans toutes leurs circonstances. (Retz, 1987, II, p. 282-283)

Cette «infinité d'occasions », ce sont ces instants que doivent analyser les hommes d'action lorsqu'ils sont au cœur des évènements ${ }^{3}$ : même eux, qui les connaissent le mieux, échouent bien souvent à les cerner. Il n'y a rien d'étonnant, dès lors, à lire que les « misérables gazetiers de ce temps-là ont forgé [...] des contes de Peaux d'ânes plus ridicules que ceux que l'on fait aux enfants. »(Retz, 1987, II, p. 116) Si même ceux qui ont vécu les évènements ont pris l'apparence pour la réalité, on comprend pourquoi Retz s'indigne de ces «auteurs impertinents qui, étant nés dans la basse-cour et n'ayant jamais passé l'antichambre, se piquent de ne rien ignorer de tout ce qui s'est passé dans le cabinet. » (Retz, 1987, II, p. 159) On peut d'ailleurs mesurer à quel point Retz avance une conception unique de l'histoire et de

\footnotetext{
${ }^{2}$ Comme le soulignent André Bertière (p. 210) et Malina Stefanovska (p. 134).

${ }^{3}$ Instant et occasion, tout comme le moment dont il est souvent question dans les Mémoires, sont des notions

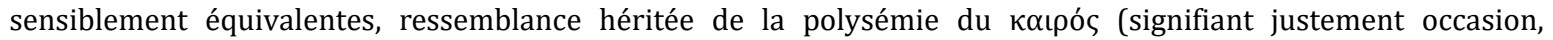
moment opportun, ou pouvant désigner simplement une brève unité de temps). L'instant et le moment deviennent occasion grâce à la perspicacité de l'observateur confronté au «fourmillement des possibles au cœur de chaque instant» (Garapon, 2011, p. 130). Comme l'affirme également Stefanovska: «Fascinated by the volatily of circumstances, Retz thus presents himself as a virtuoso and a theorist of the opportune moment, the so-called kairos of Hellenistic culture.» («Fasciné par la volatilité des circonstances, Retz se présente ainsi comme un virtuose et un théoricien du moment opportun, le kairos des Grecs anciens. » [Je traduis]; p. 189).
} 
l'historiographie en rappelant que les théoriciens du genre au XVII siècle, sous l'influence de la rhétorique classique, refusaient la présence de l'invraisemblable dans des textes ayant pourtant pour objectif de raconter la vérité (Guion, 2010, p. 24-25).

Une anecdote de la première partie des Mémoires est particulièrement éclairante pour comprendre la complexité de l'instant et à quel point il est difficile de l'interpréter correctement ${ }^{4}$. Après une nuit de fête et de théâtre, le carrosse dans lequel le cardinal de Retz et quelques autres avançaient s'est immobilisé, et l'on aperçoit au loin ce qui s'apparente à des « diables» (Retz, 1987 I, p. 252). Retz et Henri de la Tour d'Auvergne, vicomte de Turenne, osent s'approcher, l'épée à la main, pour constater que ces «fantômes » (Retz, 1987, I, p. 253) n'étaient en fait que des capucins en procession :

Tout cela se passa, comme vous vous pouvez imaginer, en même temps et en moins de rien. [...] Nous retournâmes au carrosse, M. de Turenne et moi, avec les éclats de rire que vous vous pouvez imaginer, et nous fîmes, lui et moi, dès le moment même, deux observations, que nous nous communiquâmes dès le lendemain matin. Il me jura que la première apparition de ces fantômes imaginaires lui avait donné de la joie, quoiqu'il eût toujours cru auparavant qu'il aurait peur s'il voyait jamais quelque chose d'extraordinaire; et je lui avouai que la première vue m'avait ému, quoique j'eusse souhaité toute ma vie de voir des esprits. La seconde observation que nous fîmes fut que tout ce que nous lisons dans la vie de la plupart des hommes est faux. M. de Turenne me jura qu'il n'avait pas senti la moindre émotion, et il convint que j'avais eu sujet de croire, par son regard si fixe et par son mouvement si lent, qu'il en avait eu beaucoup. Je lui confessai que j'en avais eu d'abord, et il me protesta qu'il aurait juré sur son salut que je n'avais eu que du courage et de la gaieté. Qui peut donc écrire la vérité, que ceux qui l'ont sentie? Et le président de Thou a eu raison de dire qu'il n'y a de véritables histoires que celles qui ont été écrites par les hommes qui ont été assez sincères pour parler véritablement d'eux-mêmes. (Retz, 1987, I, p. 253-254)

En plus du problème de la perception de la réalité - avoir pris des capucins pour des fantômes -, l'interprétation de l'anecdote met en lumière une complexité bien plus décisive : Retz et de Turenne ont compris le contraire de ce qui animait véritablement l'autre, et chacun a réagi à l'inverse complet de ce à quoi ils s'attendaient d'eux-mêmes, alors qu'ils n'essayaient

\footnotetext{
${ }^{4}$ Cette anecdote est partiellement fausse, car le cardinal n'était pas présent lorsqu'elle s'est produite, comme le souligne Hubert Carrier dans «Sincérité et création dans les Mémoires du cardinal de Retz » (p. 101). L’important est le rôle que lui attribue Retz dans ses Mémoires, et c'est ce point de vue que nous entendons restituer.
} 
pas de se duper mutuellement, ce qui est souvent le cas entre ennemis dans une guerre civile. Ainsi, parler véritablement de soi-même ${ }^{5}$, par opposition aux discours des gazetiers et historiens, c'est admettre que le jugement et l'intelligence ont peu de prises dans l'immédiateté de l'évènement, que le présent et le passé sont bien souvent illisibles, car trop denses à interpréter, et que tout est trompeur, tant par les faiblesses de l'esprit humain que cet exemple vient d'illustrer que par l'habitude : « Nous nous accoutumons à tout ce que nous voyons; et je vous ai dit quelquefois que je ne sais si le consulat du cheval de Caligula nous aurait autant surpris que nous nous l'imaginons. » (Retz, 1987, I, p. 380) Comme le remarque Delphine Mouquin de Garidel, dans l'anecdote des capucins, «[1]a peur pousse à faire l'épreuve du réel » (p. 288) et révèle, dans une réflexion introspective du mémorialiste, sa complexité. Or, dans le chaos d'une guerre civile, les moments de ce genre abondent :

Je me confirme dans ce que j'ai dit ci-devant, qu'il y a des points dans les affaires qui échappent, par des rencontres même naturelles, aux plus clairvoyants, et que nous en rencontrerions bien plus fréquemment dans les histoires, si elles étaient toutes écrites par des gens qui eussent été eux-mêmes dans le secret des choses. (Retz, 1987, II, p. 159)

Et il ajoute ailleurs : «il y a des points inexplicables dans les affaires et inexplicables même dans leurs instants » (Retz, 1987, II, p. 154).

Enfin, le monde s'opacifie dans les Mémoires du cardinal de Retz non seulement parce que les moments décisifs sont souvent incompréhensibles, mais aussi parce qu'ils ne s'agencent pas entre eux. Les hommes d'action agissent de manière contradictoire, dans des instants qu'ils interprètent mal et qu'il est impossible de lier :

Un historien qui décrirait des temps qui seraient plus éloignés de son siècle chercherait des liaisons à des incidents aussi peu vraisemblables et aussi contradictoires, si l'on peut parler ainsi, que sont ceux-là. Il n'y eut pas plus

\footnotetext{
5 Et c'est en ce sens que l'anecdote des capucins est vraie : non pas en ce qu'elle narre un évènement réel, mais en ce que c'est le cardinal lui-même qu'elle permet de connaître. Au sujet de cette anecdote et de la manière dont nous nous trouvons à justifier le mensonge du cardinal, nous renvoyons le lecteur à l'article de Frédéric Briot sur lequel nous nous sommes appuyé : «Comment croire les mémorialistes sur parole? » (p.53-64), et à celui de Hubert Carrier (p. 95-116), mentionné ci-haut.
} 
d'intervalle que celui que je vous ai marqué entre les uns et les autres; il n'y eut pas plus de mystère. Tout ce que les politiques du vulgaire se sont voulu figurer, pour concilier ces événements, n'est que fiction, n'est que chimère. J'en reviens toujours à mon principe, qui est que les fautes capitales font, par des conséquences presque inévitables, que ce qui paraît et est en effet le plus étrange et le plus extravagant est possible. (Retz, 1987, II, p. 412-413)

En effet, après avoir présenté ce qui l'a mené à obtenir la coadjuterie de Paris (une importante fonction ecclésiastique qui le destinait à devenir archevêque de la ville), Retz se demande : « [q]uel rapport de ces deux bagatelles à l'archevêché de Paris? et voilà toutefois comme la plupart des choses se font. » (Retz, 1987, I, p. 261) Il n'y avait donc rien à justifier, et le hasard a fait en sorte qu'un mot d'esprit et une bonne impression laissée à Louis XIII l'ont mené à cette charge importante. L'historien et les gazetiers auraient donc la tâche impossible - s'ils n'étaient pas si vains - de rendre compte de cette infinité d'occasions, cette succession d'« instants hétérogènes » (Garapon, 2005, p. 120) où être et paraître sont inextricablement emmêlés. Agir dans le présent et comprendre le passé sont d'une égale complexité, et le passé est difficile à saisir parce que le présent même l'était: l'homme d'action du présent, tout comme l'historien, est plongé dans un épais brouillard d'apparences trompeuses, et il doit agir lors d'évènements « bizarres, rares, extraordinaires » (Retz, 1987, II, p. 371).

\section{Une solution partielle au problème de l'action: les maximes et les portraits}

Or comment agir comme il se doit dans ces conditions, dans une période aussi troublée et cruciale pour le sort d'un royaume qu'une guerre civile? Comment savoir à qui se fier et de qui il faut se défier, ce qui est nécessaire dans la conduite d'un parti, mais aussi lors des innombrables négociations avec les membres du camp adverse? Au brouillard de l'instant - la

brièveté temporelle - répond dans les Mémoires la brièveté formelle : d'une part les maximes, 
sentences et réflexions ${ }^{6}$, tout autant de phrases bien tournées qui restent dans la mémoire et offrent des règles, des conseils de conduite ou des observations variées; d'autre part les portraits qui révèlent au grand jour le cœur de l'être humain pour celui qui prend le soin de l'observer, de voir le mouvement ou l'inquiétude qui le trahit. L'apparente simplicité de ces procédés qui, placés au détour d'une phrase, passent presque inaperçus, cache toutefois une subtilité, voire une ambiguïté. En effet, là où le lecteur croit se saisir d'éléments stables à travers l'inconstance des choses mondaines, ces formes brèves ne demeurent que des solutions partielles au problème de l'action tel que présenté dans les Mémoires : ancrées dans un monde aussi complexe, elles ne peuvent, pour cette même raison, en venir à bout.

Comme l'a bien montré André Bertière, les maximes de Retz prennent forme dans l'expérience. Leur auteur a le "goût des nuances »- on se serait en effet étonné de le voir simplifier la réalité -, mais il résiste à tout «verbalisme » (Bertière, 1977, p. 500) et ce n'est pas le plaisir du jeu mondain qui l'intéresse dans cette forme brève. De même que c'est son rôle d'acteur dans les évènements qui permet à Retz de les écrire - contrairement aux historiens et gazetiers -, c'est à partir de l'action qu'il a déduit ses maximes. Des formules telles que « [j]'avais eu mille fois cette vue » (Retz, 1987, I, p. 315), «j'ai observé mille fois », (Retz, 1987, I, p. 354, 540), «plus de mille fois cette réflexion » (Retz, 1987, I, p. 379-380) ou «mille et mille fois » (Retz, 1987, I, p. 337), ou plus modestement « j'ai fait cette observation trois ou quatre fois en ma vie » (Retz, 1987, II, p. 169), ou encore « [j]e connus à cet instant que [...] » (Retz, 1987, I, p. 370), précèdent ses maximes de manière régulière et fondent leur autorité en prouvant d'emblée leur efficacité.

Ainsi, certaines de ses maximes sont limpides («J'ai observé mille fois qu'il est aussi nécessaire de choisir les mots dans les grandes affaires, qu'il est superflu de les affecter dans

\footnotetext{
${ }^{6}$ Nous employons ici sentences, maximes et réflexions sans trop les distinguer, puisque comme l'affirme Simone Bertière : «Peu importe à Retz. » (Retz, 1991, p. 13)
} 
les petites » [Retz, 1987, I, p. 540]), et indiquent clairement comment agir : « Le grand secret de ceux qui entrent dans les emplois est de saisir d'abord l'imagination des hommes par une action que quelque circonstance leur rende particulière. » (Retz, 1987, I, p. 263) Elles donnent par exemple des stratégies pour réussir son ascension : «j'éprouvai [...] que descendre jusques aux petits est le plus sûr moyen pour s'égaler aux grands » (Retz, 1987, I, p. 264). Retz se permet aussi de confirmer celles d'autres hommes d'action : «Le vieux prince d'Orange disait que le moment où l'on recevait les plus grandes et les plus heureuses nouvelles était celui où il fallait redoubler son attention pour les petites. » (I, p. 412) Il n’hésite pas non plus à enseigner la dissimulation :

Il n'est jamais permis à un inférieur de s'égaler en parole à celui à qui il doit du respect, quoiqu'il s'y égale dans l'action; et il l'est aussi peu à un ecclésiastique de confesser qu'il est armé, même quand il l'est. Il y a des matières sur lesquelles il est constant que le monde veut être trompé. (Retz, 1987, II, p. 228)

Mais devant l'opacité du monde, il est normal que certaines maximes se montrent ambiguës à leur tour. C'est le cas de la maxime suivante qui implique déjà que l'on se connaisse suffisamment pour ne pas se duper soi-même en se percevant plus apte que dans la réalité : « il n'y a rien de plus malhabile que de se faire croire capable des choses dont les exemples sont à craindre» (Retz, 1987, I, p. 237). Elle implique également d'avoir une expérience suffisante des choses politiques pour lier ses projets à d'autres, et reconnaître leur fragilité selon les exemples qui servent au rapprochement. En effet, Retz prévient ses lecteurs qu'« [i]l n'est pas étrange que les hommes ne se connaissent pas : il y a des temps où l'on peut dire même qu'ils ne se sentent point » (Retz, 1987, II, p. 359). Par ailleurs, alors que tout devrait nous inciter à nous montrer sur nos gardes dans ce monde trompeur, cette attitude même peut nous déjouer: «[c]ette considération est une de celles qui m’ont obligé de vous dire quelquefois que l'on est plus souvent dupe par la défiance que par la confiance » (Retz, 1987, I, 
p. 282). Retz réécrit qu'il «connu[t] encore en cette occasion que les gens les plus défiants sont souvent les plus dupes » (Retz, 1987, II, p. 461). Enfin, l'incertitude est telle que même la bonne conduite peut entraîner de mauvaises répercussions : «Voilà le malheur des guerres civiles : l'on y fait souvent des fautes par bonne conduite. » (Retz, 1987, I, p. 446)

Il en va de même des portraits, qui rejoignent les maximes en ce que ceux-ci se fondent bien souvent sur l'observation psychologique, type d'observation nécessaire dans la mesure où les gens mentent :

La vérité est que tout ce qui était dans ce cabinet jouait la comédie : je faisais l'innocent, et je ne l'étais pas, au moins en ce fait; le Cardinal faisait l'assuré, et il ne l'était pas si fort qu'il le paraissait; il y eut quelques moments où la Reine contrefit la douce, et elle ne fut jamais plus aigre; M. de Longueville témoignait de la tristesse, et il était dans une joie sensible, parce que c'était l'homme du monde qui aimait le mieux les commencements de toutes affaires; [...] le maréchal de Villeroy faisait le gai pour faire sa cour au ministre, et il m'avouait en particulier, les larmes aux yeux, que l'État était sur le bord du précipice [...]. (Retz, 1987, I, p. 306)

Si certains êtres sont aisément pénétrables ${ }^{7}$, d'autres sont fortement marqués par l'ambivalence. Sensible au détail qui trahit les individus ${ }^{8}$, Retz n'est pas moins conscient que ceux-ci peuvent se montrer contradictoires et posséder « une part irréductible d'irrationnel » (Bertière, 1977, p. 206). Chez certains, et non les moindres, il reste une part d'incompréhensible, et en cela le mémorialiste tranche à nouveau avec son siècle, qui « est à la recherche d'une psychologie cohérente, qui rende intelligibles, voire prévisibles, à partir des données initiales de son caractère, les réactions d'un individu » (Bertière, 1977, p. 203). Il voit parfois chez les hommes « un certain air [...] qui ne se peut exprimer, mais qui prouve [leurs intentions] souvent beaucoup mieux que tout ce qui se peut expliquer » (Retz, 1987, II, p. 154).

\footnotetext{
7 C'est le cas de la régente Anne d'Autriche (Retz, 1987, I, p. 371), comme en témoigne son portrait d'une redoutable efficacité et remarquablement analysé par Patrick Dandrey (p. 26-33).

${ }^{8}$ Par exemple : «Comme l'on ne se peut jamais si bien contraindre qu'il n'échappe toujours quelque chose du naturel, il [un cardinal, lors du conclave auquel Retz a participé] ne se put si bien couvrir que je ne m'aperçusse qu'il était homme de minuties : ce qui est toujours signe non pas seulement d'un petit génie, mais encore d'une âme basse. Il me parlait un jour des études de sa jeunesse, et il me disait qu'il avait été deux ans à écrire d'une même plume. Cela n'est qu'une bagatelle; mais comme j'ai remarqué plusieurs fois que les plus petites choses sont souvent de meilleures marques que les plus grandes, cela ne me plut pas. » (Retz, 1987, II, p. 505)
} 
Le portrait tout en nuances de Richelieu exemplifie aussi cette caractéristique de l'art retzien du portrait, ce ministre étant dépeint par un savant équilibre de défauts et de qualités : «il faut confesser que tous ses vices ont été de ceux que la grande fortune rend aisément illustres, parce qu'ils ont été de ceux qui ne peuvent avoir pour instruments que de grandes vertus » (Retz, 1987, I, p. 287). Jacqueline Plantié, dans son incontournable Mode du portrait littéraire en France (1641-1681), a mis en lumière comment Retz « respecte, et même [...] met volontiers en valeur, l'ambiguïté d'un être. Il s'arrête au seuil de l'inexplicable, il reconnaît que l'intelligence est impuissante pour comprendre totalement une personne » (p. 651). D'ailleurs, Retz lui-même n'échappe pas à cette difficulté : «l'on ne se connaît jamais assez bien pour se peindre raisonnablement soi-même » (Retz, 1987, I, p. 377), affirme-t-il pour justifier l'absence de son propre portrait dans la galerie des principaux acteurs de la Fronde. C'est en fournissant des clefs de compréhension des êtres et des évènements que les maximes et les portraits peuvent partiellement résoudre la manière dont il faut agir dans le monde tout en soulignant à nouveau son obscurité.

\section{Résolution et occasion : le retour de la brièveté temporelle}

Cela étant dit, cette conception de l'histoire et le problème de l'action qui lui est rattachée ne se résolvent pas dans une forme de défaitisme et n'acculent pas le chef de parti à la paralysie. Si l'homme plongé dans les conflits de son temps ne peut s'assurer la victoire, il peut encore s'illustrer - plus qu'échouer - dans l'honneur, gloire à laquelle se raccroche Retz dans ses Mémoires. La capacité à bien agir, et surtout à voir les occasions dans l'adversité révèle la grandeur, et tant s'en faut qu'elle soit hors d'atteinte.

En effet, Mazarin a peut-être vaincu les frondeurs, mais sans gloire, tandis que Retz agissait d'une manière bien plus noble et élevée à ses yeux : «Les plus grands dangers ont 
leurs charmes pour peu que l'on aperçoive de gloire dans la perspective des mauvais succès; les médiocres n'ont que des horreurs quand la perte de la réputation est attachée à la mauvaise fortune. » (Retz, 1987, I, p. 327) Il écrit aussi que «l'on doit hasarder le possible toutes les fois que l'on se sent en état de profiter même du manquement de succès » (Retz, 1987, II, p. 68), que « [l]es extrêmes sont toujours fâcheux; mais [qu']ils sont sages quand ils sont nécessaires. Ce qu'ils ont de consolatif est qu'ils ne sont jamais médiocres et qu'ils sont décisifs quand ils sont bons » (Retz, 1987, I, p. 327-328). Puisque la guerre civile est une période où l'extraordinaire peut se produire, il faut savoir prendre des risques : « Il n'y a rien dans le monde qui n'ait son moment décisif, et le chef-d'œuvre de la bonne conduite est de connaître et de prendre ce moment. » (Retz, 1987, I, p. 339) Il faut non seulement voir, mais surtout saisir l'occasion : « il y a des feux qui embrasent tout : l'importance est d'en connaître et d'en prendre le moment » (Retz, 1987, I, p. 244). Or il est inutile d'espérer bien agir sans la sagacité entreprenante qui distingue les grands hommes, au contraire de certains acteurs de la guerre civile :

Monsieur le Comte avait toute la hardiesse du cœur que l'on appelle communément vaillance, au plus haut point qu'un homme la puisse avoir; et il n'avait pas, même dans le degré le plus commun, la hardiesse de l'esprit, qui est ce que l'on nomme résolution. La première est ordinaire et même vulgaire; la seconde est même plus rare que l'on ne se le peut imaginer : elle est toutefois encore plus nécessaire que l'autre pour les grandes actions; et y a-t-il une action plus grande au monde que la conduite d'un parti? Celle d'une armée a, sans comparaison, moins de ressorts, celle d'un État en a davantage; mais les ressorts n'en sont, à beaucoup près, ni si fragiles ni si délicats. Enfin je suis persuadé qu'il faut de plus grandes qualités pour former un bon chef de parti que pour faire un bon empereur de l'univers; et que dans le rang des qualités qui le composent, la résolution marche du pair avec le jugement : je dis avec le jugement héroïque, dont le principal usage est de distinguer l'extraordinaire de l'impossible. Monsieur le Comte n'avait pas un grain de cette sorte de jugement, qui ne se rencontre même que très rarement dans un grand esprit, mais qui ne se trouve jamais que dans un grand esprit. (Retz, 1987, I, p. 238-239)

Si André Bertière a bien montré l'effritement progressif de la figure du héros dans les Mémoires du cardinal de Retz (p. 323-392), son ombre ne plane pas moins sur le récit dans un 
habile « composé de Plutarque et de Machiavel » (p. 380) - du Corneille du Cid et du jésuite espagnol Balthasar Gracián , pourrait-on aussi ajouter -, et cette possibilité d'un jugement ou d’une prévoyance qui semble dépasser les capacités humaines demeure malgré tout: «Je ne sais si je n'ai point déjà dit, en quelque endroit de cet ouvrage, que ce qui a le plus distingué les hommes est que ceux qui ont fait les grandes actions ont vu devant les autres le point de leur possibilité. » (Retz, 1987, II, p. 424)

Remarquons aussi dans ce portrait de Monsieur le Comte l'importance de la résolution, et son corollaire, la condamnation de l'irrésolution qui marque le caractère des hommes qui ne sont pas faits pour l'action. Cette résolution semble même un préalable au «jugement héroïque » mentionné ci-dessus. Il faut d'abord et avant tout choisir, ne pas être paralysé par l'indécision : «J'ai déjà remarqué, en quelque endroit de cet ouvrage, que tout ce qui est interlocutoire paraît sage aux esprits irrésolus, parce que leur inclination les portant à ne point prendre de résolution finale, ils flattent d'un beau titre leur propre sentiment. » (Retz, 1987, II, p. 67) De même, « les esprits irrésolus ne suivent presque jamais ni leur vue ni leur sentiment, tant qu'il leur reste une excuse pour ne pas se déterminer » (Retz, 1987, II, p. 99). À l'inverse, le moment où le cardinal de Retz décide de s'opposer à la cour et d'entrer dans la fronde parlementaire est un exemple de résolution, de capacité à oser prendre des risques, ce préalable de la grandeur :

Comme ils m'eurent laissé tout seul pour le quart d'heure que je leur avais demandé, je ne fis pas seulement réflexion sur ce que je pouvais, parce que j'en étais très assuré : je pensai seulement à ce que je devais, et je fus embarrassé. Comme la manière dont j'étais poussé et celle dont le public était menacé eurent dissipé mon scrupule, et que je crus pouvoir entreprendre avec honneur et sans être blâmé, je m'abandonnai à

\footnotetext{
${ }_{9}^{9}$ Dans un article sur la pointe baroque, Jean-Vincent Blanchard écrit, au sujet des maximes de Gracián, cette phrase qui pourrait aussi bien s'appliquer à Retz : « Dans ce hasard relatif, l'art tient à saisir la bonne occasion, le moment où les choses sont à point. Alors, dans le temps cyclique des empires vient s'insérer le temps au cours duquel s'exerce la prudence du particulier, au moment de la performance. La brièveté s'invente constamment pour sonder les multiples aspects d'une réalité historique en constant changement.» (p.354) Telle est en effet l'une des fonctions des maximes de Retz : enseigner à saisir l'occasion dans une réalité ambiguë.
} 
toutes mes pensées. Je rappelai tout ce que mon imagination m'avait jamais fourni de plus éclatant et de plus proportionné aux vastes desseins; je permis à mes sens de se laisser chatouiller par le titre de chef de parti, que j'avais toujours honoré dans les Vies de Plutarque; mais ce qui acheva d'étouffer tous mes scrupules fut l'avantage que je m'imaginai à me distinguer de ceux de ma profession par un état de vie qui les confond toutes. [...] Le souper du Palais-Royal et la résolution de me perdre avec le public l'ayant purifiée [son hésitation], je la pris avec joie [la vue de devenir chef de parti], et j'abandonnai mon destin à tous les mouvements de la gloire. (Retz, 1987, I, p. 315; nous soulignons)

En un «quart d'heure », Retz prend le risque - il s'abandonne ${ }^{10}$ - et se résout à entrer sur le théâtre de la guerre civile en étant pleinement conscient de la fin incertaine qui l'attendait. Il s'agit désormais de constamment chercher l'occasion et ne plus cesser d'entreprendre des démarches même quand la situation semble désespérée :

L'un des plus grands défauts des hommes est qu'ils cherchent presque toujours, dans les malheurs qui leur arrivent par leurs fautes, des excuses devant que d'y chercher des remèdes; ce qui fait qu'ils y trouvent très souvent trop tard les remèdes, qu'ils n'y cherchent pas d'assez bonne heure. (Retz, 1987, I, p. 455)

À l'inverse, « rien ne marque tant le jugement solide d'un homme, que de savoir choisir entre les grands inconvénients» (Retz, 1987, I, p. 395). Ceux qui balancent sans se décider ne peuvent affronter les périls et redresser la situation par une action qui semblait impossible tant qu'elle n'avait pas été tentée : «Il me semble que je vous ai déjà dit ailleurs que tout ce qui est fort extraordinaire ne paraît possible, à ceux qui ne sont capables que de l'ordinaire, qu'après qu'il est arrivé. » (Retz, 1987, II, p. 463) Le Prince de Condé a illustré ce principe lors de la bataille de Lens, quelques mois avant la guerre civile : «Je ne me puis empêcher de vous dire que le combat étant presque perdu, Monsieur le Prince le rétablit et le gagna par un seul

\footnotetext{
10 Une telle attitude implique le courage de prendre des risques. Cette dernière s'oppose à la peur qui porte les hommes à se laisser déterminer par les autres, à ne rien entreprendre que dans l'aveuglement que leur induit leur irrésolution: «Monsieur faisait, en toutes choses, comme font la plupart des hommes quand ils se baignent : ils ferment les yeux en se jetant dans l'eau. » (Retz, 1987, II, p. 67) Permettons-nous aussi d'ajouter que l'inaction ne relève pas nécessairement de l'irrésolution, même si elle est exceptionnelle dans les Mémoires : « Je vous vous ai dit ci-dessus que j'avais fait la résolution de demeurer tout le plus qu'il me serait possible dans l'inaction, parce qu'il est vrai que j'avais beaucoup à perdre et rien à gagner dans le mouvement. J'accomplis, en partie, cette résolution, parce qu'il est vrai que je n'entrai presque en rien de tout ce qui se fit en ce temps-là, étant très convaincu qu'il n'y avait rien de bon à faire pour l'ordinaire. » (Retz, 1987, II, p. 339) Une inaction peut être résolue.
} 
coup de cet œil d'aigle que vous lui connaissez, qui voit tout dans la guerre et qui ne s'y éblouit jamais. » (Retz, 1987, I, p. 300)

Enfin, ce prince souligne aussi que la grandeur et l'honneur peuvent se conserver même dans la défaite. En concluant le portrait qui lui est dédié, Retz écrit : « Il n’a pu remplir son mérite, c'est un défaut; mais il est rare, mais il est beau » (Retz, 1987, I, p. 372). De fait, les défauts et les fautes des hommes peuvent aussi signaler leur grandeur puisqu'« il y a certains défauts qui marquent plus une bonne âme que de certaines vertus » (Retz, 1987, I, p. 486) :

La même vérité qui m'oblige à remarquer la faute m'oblige à en admirer le principe; et il est si beau à l'homme du monde du courage le plus héroïque d'avoir péché par excès de douceur, que ce qui ne lui [a] pas succédé dans la politique, doit être au moins admiré et exalté par tous les gens de bien dans la morale. (Retz, 1987, II, p. 335)

Une action juste est préférable au calcul politique consommé, qui constitue une ruse des plus basses. La dissimulation, qui est nécessaire - nous l'avons observé dans certaines maximes n'en a pas moins ses limites. Comme le signale Marc Fumaroli, « Retz veut la gloire, non le succès » (p. 264), ou du moins il préfère s'accommoder de la première que de la perdre au prix de la seconde. La dissimulation doit être au service de la grandeur et de la gloire, et c'est en ce sens qu'elle fait partie « de l’attitude héroïque telle que Balthasar Gracián l'a définie à la suite de Machiavel et de Tacite» (Merlin-Kajman, 2012, p. 72) et que Retz reprend à son compte.

En dernière analyse, Retz ne prétend pas échapper à l'opacité de l'histoire et du présent, cette succession d'instants indéchiffrables et soumis au hasard que les gazetiers et historiens ont eu la vanité d'essayer de décrypter. Ses maximes peuvent orienter l'homme d'action, mais lui enseignent surtout cette ambigüité dans laquelle il est plongé. De même, le portrait, dressé à partir des fines observations psychologiques, permet de comprendre le caractère d'un être, tout en ayant la modestie de ne pas en faire une caricature. Il y a une part 
d'inexplicable dans les évènements et les instants, tout comme dans le cœur humain. La grandeur, dans ces conditions, consiste d'une part à se montrer résolu en saisissant les occasions qui se présentent et même, d'autre part, à créer ces occasions en agissant constamment. Dans cette perspective où il est impossible de se garantir la victoire, c'est l'honneur qui doit triompher, quitte à ce que cela se produise dans la défaite : « je [...] répondis qu'il était vrai que nous hasardions notre vie; mais que si [nous] agissions autrement, nous perdrions certainement notre honneur » (Retz, 1987, I, p. 533). Les Mémoires, ancrés dans l'expérience, font revivre au lecteur cette agitation quand Retz emploie, à l'occasion, des formules telles que : "J'ai couru jusques ici à perte d'haleine sur ces matières » (Retz, 1987, I, p. 294), ou encore quand il affirme qu'il raconte les faits « en courant » (Retz, 1987, I, p. 291). Le récit incarne la leçon que la conduite de Retz a tenté de respecter toute sa vie, soit de se remuer constamment, pour paraphraser Tallemant des Réaux ${ }^{11}$, s'agiter sans cesse pour voir l'occasion et savoir s'y abandonner.

\section{Bibliographie}

BERTIÈRE, André (1977), Le cardinal de Retz mémorialiste, Paris, Klincksieck.

BLANCHARD, Jean-Vincent (2002), « Raison d'État présent : vers une phénoménologie politique de la pointe baroque », dans Erec R. Koch (dir.), Classical Unities: Place, Time, Action. Actes du $32^{e}$ congrès annuel de la North American Society for Seventeenth-Century French Literature, Tübingen, Narr, coll. « Biblio 17 », p. 347-357.

BRIOT, Frédéric (1995), « Comment croire les mémorialistes sur parole? », Revue des Sciences humaines, no 238, p. 53-64.

CARRIER, Hubert (2005), "Sincérité et création littéraire dans les Mémoires du cardinal de Retz », dans Pierre Ronzeaud (dir.), Cardinal de Retz. Mémoires. Anthologie critique, Paris, Klincksieck, coll. « Parcours critique », p. 91-116.

\footnotetext{
11 « Il a toujours été [le cardinal de Retz] d'humeur remuante. » (Tallemant des Réaux, cité par Stefanovska, 2002, p. 35)
} 
Charbonneau, Frédéric (2001), Les silences de l'histoire. Les mémoires français du XVIIe siècle, Québec, Presses de l’Université Laval, coll. «République des Lettres : études ».

DANDREY, Patrick (2005), «Commentaire comparé de deux portraits tirés des Mémoires du cardinal de Retz», dans Pierre Ronzeaud (dir.), Cardinal de Retz. Mémoires. Anthologie critique, Paris, Klincksieck, coll. « Parcours critique », p. 203-216.

Fumaroli, Marc (2002), "Retz: des Mémoires en forme de conversation galante », dans La diplomatie de l'esprit. De Montaigne à La Fontaine, Paris, Gallimard, coll. « Tel », p. 247-281.

GARAPON, Jean (2005), " Les vérités diverses des Mémoires du cardinal de Retz », dans Pierre Ronzeaud (dir.), Cardinal de Retz. Mémoires. Anthologie critique, Paris, Klincksieck, coll. «Parcours critique », p. 117-125.

GARAPON, Jean (2011), "Curiosité et écriture dans les Mémoires du cardinal de Retz », dans Jean Garapon et Christian Zonza (dir.), Nouveaux regards sur les Mémoires du Cardinal de Retz. Actes du colloque organisé par l'Université de Nantes, Tübingen, Narr, coll. «Biblio $17 »$, p. 123-133.

GuION, Béatrice (2010), "Comment écrire l'histoire : l'ars historica à l'âge classique », XVIIe siècle, no 246 , p. 9-25.

LESNE-JAFFRO, Emmanuèle (2006), "Les Mémoires : une critique de l'histoire au XVII siècle ", dans Sabrina Vervacke, Éric Van der Schueren et Thierry Belleguic (dir.), Les songes de Clio. Fiction et Histoire sous l'Ancien Régime, Québec, Presses de l'Université Laval, coll. «République des Lettres », p. 487-510.

MERLIN-KAJMAN, Hélène (2012), «Extraordinaire, extrémisme et modération dans les Mémoires du Cardinal de Retz », La Licorne, no 99, p. 67-82.

MouQuin-DE GARIDEL, Delphine (2016), «Au fantôme! Quelques revenants dans les Mémoires de la Grande Mademoiselle à Chateaubriand», dans Christian Zonza (dir.), Vérités de l'histoire et vérité du moi. Hommage à Jean Garapon, Paris, Honoré Champion, coll. «Colloques, congrès et conférences sur le Classicisme », p. 281-296.

PlantiÉ, Jacqueline (1994), La mode du portrait littéraire en France (1641-1681), Paris, Honoré Champion, coll. « Lumière classique».

RETz, Paul de Gondi cardinal de (1987), Mémoires, édition de Simone Bertière, Paris, Garnier, coll. «Classiques Garnier », 2 v.

RETZ, Paul de Gondi cardinal de (1991), Maximes et réflexions, édition de Simone Bertière, Paris, Éditions de Fallois.

STEFanovsKa, Malina (2002), « Démarches et itinéraires de la Fronde : Paris dans les Mémoires du cardinal de Retz », dans Erec R. Koch (dir.), Classical Unities: Place, Time, Action. Actes du 
$32^{e}$ congrès annuel de la North American Society for Seventeenth-Century French Literature, Tübingen, Narr, coll. « Biblio 17 », p. 35-44.

Stefanovska, Malina (2008), La politique du cardinal de Retz. Passions et factions, Rennes, Presses universitaires de Rennes, coll. «Interférences ».

STEFAnOvSKA, Malina (2009), «Cardinal de Retz's Memoirs: Encountering Fortune and Taking Timely Steps », dans John D. Lyons et Kathleen Wine (dir.), Chance, Literature, and Culture in Early Modern France, Farnham/Burlington, Ashgate, p. 183-195.

\title{
Résumé
}

Cet article souhaite arrimer la brièveté temporelle qui découle de la conception de l'histoire des Mémoires du cardinal de Retz (une conception qui tend à un effritement temporel composé d'une succession d'instants impénétrables) à la brièveté formelle des maximes et réflexions qui, elle, montre en contrepartie comment agir dans un monde opaque. Cependant, se refusant à la simplification, les Mémoires font voir que cette obscurité du monde et du temps ne peut être vaincue. La recherche de la victoire politique est alors sublimée par celle de l'honneur et de la gloire qui, elle, peut s'accommoder de la défaite.

\begin{abstract}
This article seeks to anchor the temporal brevity that results from cardinal de Retz's conception of history as developed in his Mémoires (a conception that leads to a desintegration of time in a succession of impenetrable moments) into the formal brevity of the maxims and portraits that, in counterpart, show how to act in an opaque world. However, since Retz refuses simplification, the Mémoires also show that the opacity of the world cannot be overthrown: the search for political victory is therefore sublimated in the search for honor and glory, even if they are found in defeat.
\end{abstract}

